\title{
Exploring the Very Extended Low-surface-brightness Stellar Populations of the Large Magellanic Cloud with SMASH
}

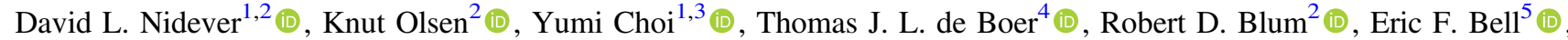 \\ Dennis Zaritsky ${ }^{3}$ (D) Nicolas F. Martin ${ }^{6,7}$ (1), Abhijit Saha ${ }^{2}$, , Blair C. Conn ${ }^{8,9}$ (i), Gurtina Besla ${ }^{3}$, Roeland P. van der Marel ${ }^{10}$ (i), \\ Noelia E. D. Noël ${ }^{4}$, Antonela Monachesi ${ }^{11,12}$ (1) , Guy S. Stringfellow ${ }^{13}$ (1) , Pol Massana ${ }^{4}$, Maria-Rosa L. Cioni ${ }^{14}$ (1),

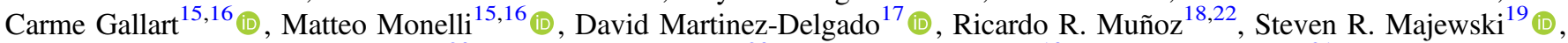 \\ A. Katherina $\operatorname{Vivas}^{20}$ (10), Alistair R. Walker ${ }^{20}$ (D), Catherine Kaleida ${ }^{10}$, and You-Hua Chu ${ }^{21}$ (1) \\ ${ }^{1}$ Department of Physics, Montana State University, P.O. Box 173840, Bozeman, MT 59717-3840, USA; dnidever@montana.edu \\ ${ }^{2}$ National Optical Astronomy Observatory, 950 North Cherry Avenue, Tucson, AZ 85719, USA \\ ${ }^{3}$ Steward Observatory, University of Arizona, 933 North Cherry Avenue, Tucson, AZ 85721, USA \\ ${ }^{4}$ Department of Physics, University of Surrey, Guildford, GU2 7XH, UK \\ ${ }^{5}$ Department of Astronomy, University of Michigan, 1085 S. University Avenue, Ann Arbor, MI 48109-1107, USA \\ ${ }^{6}$ Observatoire astronomique de Strasbourg, Université de Strasbourg, CNRS, UMR 7550, 11 rue de l'Université, F-67000 Strasbourg, France \\ ${ }^{7}$ Max-Planck-Institut für Astronomie, Königstuhl 17, D-69117 Heidelberg, Germany \\ ${ }^{8}$ Research School of Astronomy and Astrophysics, Australian National University, Canberra, ACT 2611, Australia \\ ${ }^{9}$ Gemini Observatory, Recinto AURA, Colina El Pino s/n, La Serena, Chile
${ }^{10}$ Space Telescope Science Institute, 3700 San Martin Drive, Baltimore, MD 21218, USA \\ ${ }^{11}$ Instituto de Investigación Multidisciplinario en Ciencia y Tecnología, Universidad de La Serena, Raúl Bitrán 1305, La Serena, Chile \\ 12 Departamento de Física y Astronomía, Universidad de La Serena, Av. Juan Cisternas 1200 N, La Serena, Chile \\ ${ }^{13}$ Center for Astrophysics and Space Astronomy, University of Colorado, 389 UCB, Boulder, CO 80309-0389, USA \\ ${ }^{14}$ Leibniz-Institut für Astrophysik Potsdam (AIP), An der Sternwarte 16, D-14482 Potsdam, Germany \\ ${ }^{15}$ Instituto de Astrofísica de Canarias, La Laguna, Tenerife, Spain \\ ${ }^{16}$ Departamento de Astrofísica, Universidad de La Laguna, Tenerife, Spain
${ }^{17}$ Astronomisches Rechen-Institut, Zentrum für Astronomie der Universität Heidelberg, Mönchhofstr. 12-14, D-69120 Heidelberg, Germany \\ ${ }^{18}$ Departamento de Astronomía, Universidad de Chile, Camino del Observatorio 1515, Las Condes, Santiago, Chile \\ ${ }^{19}$ Department of Astronomy, University of Virginia, Charlottesville, VA 22904, USA \\ ${ }^{20}$ Cerro Tololo Inter-American Observatory, National Optical Astronomy Observatory, Casilla 603, La Serena, Chile \\ ${ }^{21}$ Institute of Astronomy and Astrophysics, Academia Sinica, P.O. Box 23-141, Taipei 10617, Taiwan, People's Republic of China \\ Received 2018 May 4; revised 2018 December 10; accepted 2018 December 24; published 2019 March 28
}

\begin{abstract}
We present the detection of very extended stellar populations around the Large Magellanic Cloud (LMC) out to $R \sim 21^{\circ}$, or $\sim 18.5 \mathrm{kpc}$ at the LMC distance of $50 \mathrm{kpc}$, as detected in the Survey of the MAgellanic Stellar History (SMASH) performed with the Dark Energy Camera on the NOAO Blanco $4 \mathrm{~m}$ Telescope. The deep $(g \sim 24)$ SMASH color-magnitude diagrams (CMDs) clearly reveal old ( $\sim 9 \mathrm{Gyr})$, metal-poor $([\mathrm{Fe} / \mathrm{H}] \approx-0.8 \mathrm{dex})$ mainsequence stars at a distance of $\sim 50 \mathrm{kpc}$. The surface brightness of these detections is extremely low with our most distant detection at $\Sigma_{g} \approx 34 \mathrm{mag}_{\operatorname{arcsec}}{ }^{-2}$. The SMASH radial density profile breaks from the inner LMC exponential decline at $\sim 13^{\circ}-15^{\circ}$ and a second component at larger radii has a shallower slope with power-law index $\alpha=-2.2$ that contributes $\sim 0.4 \%$ of the LMC's total stellar mass. In addition, the SMASH densities exhibit large scatter around our best-fit model of $\sim 70 \%$ indicating that the envelope of stellar material in the LMC periphery is highly disturbed. We also use data from the NOAO Source catalog to map the LMC main-sequence populations at intermediate radii and detect a steep dropoff in density on the eastern side of the $\mathrm{LMC}\left(\right.$ at $\left.R \approx 8^{\circ}\right)$ as well as an extended structure to the far northeast. These combined results confirm the existence of a very extended, low-density envelope of stellar material with a disturbed shape around the LMC. The exact origin of this structure remains unclear, but the leading options include an accreted halo or tidally stripped outer disk material.
\end{abstract}

Key words: galaxies: dwarf - galaxies: halos - galaxies: individual (Large Magellanic Cloud) - Local Group Magellanic Clouds

\section{Introduction}

The advent of deep wide-area surveys in the southern hemisphere has dramatically changed our view of the size and structure of the Magellanic Clouds, particularly through the use of resolved stars as tracers of structure. Using red giant branch (RGB) and asymptotic giant branch (AGB) stars measured in the 2MASS (Skrutskie et al. 2006) and DENIS (Epchtein et al. 1997) surveys, van der Marel (2001) showed that the disk of

\footnotetext{
${ }^{22}$ Visiting astronomer, Cerro Tololo Inter-American Observatory, National Optical Astronomy Observatory, which is operated by the Association of Universities for Research in Astronomy (AURA) under a cooperative agreement with the National Science Foundation.
}

the Large Magellanic Cloud (LMC) appears both more extended, smoother, and more elongated than optical photographs suggest in these maps of intermediate-age and old stellar populations. Choi et al. (2018a) extended the reach and resolution of such maps further by using red clump stars measured by the Survey of the MAgellanic Stellar History ${ }^{23}$ (SMASH; Nidever et al. 2017) using the Dark Energy Camera (DECam; Flaugher et al. 2015) and found a distinct warp in the southwestern portion of the outer disk, $7^{\circ}$ from the center, bending $\sim 4 \mathrm{kpc}$ out of the LMC plane. In addition, Choi et al. (2018b) used the red clump maps to find a ring-like stellar

\footnotetext{
23 http://datalab.noao.edu/smash/smash.php
} 
overdensity in the LMC disk at a radius of $\sim 6^{\circ}(\sim 5.2 \mathrm{kpc})$ with an amplitude of up to $\sim 2.5$ times larger than the smooth disk.

In the LMC periphery, the Outer Limits Survey (OLS; Saha et al. 2010) used main-sequence stars as tracers along a series of probes to the north of the LMC, and found LMC populations out to at least $16^{\circ}$ radius. More recently, Mackey et al. (2016) discovered a spur-like structure in the northern LMC extending from $\sim 13^{\circ}$ to $\sim 16^{\circ}$, pointing toward the Carina dwarf spheroidal galaxy. Mackey et al. (2018) used new DECam data in the southern and southwestern region of the LMC to map out the extended stellar populations in these regions using main-sequence stars, and found a smooth LMC disk population extending to $\sim 10^{\circ}$ but with two spurs extending to $\sim 14^{\circ}$. One of these spurs is cospatial with the old RR Lyrae bridge discovered by Belokurov et al. (2017) and likely has a tidal origin, which is distinct from the Magellanic Bridge (e.g., Noël et al. 2013; Carrera et al. 2017). At a larger radius still, Muñoz et al. (2006) discovered a kinematically "cold" population of high-velocity stars in the foreground of the Carina dwarf spheroidal galaxy that were consistent with having an LMC origin but $\sim 22^{\circ}$ from the LMC center. The MAgellanic Periphery Survey (MAPS) was conducted as a follow-up of this discovery to ascertain the origin and structure of this new stellar population (Majewski et al. 2009; Nidever 2009). It confirmed the existence of the extended stellar population and mapped it across an azimuthal range of over $180^{\circ}$. Belokurov \& Koposov (2016) used blue horizontal branch (BHB) stars in Dark Energy Survey (DES; Dark Energy Survey Collaboration et al. 2016) data to discover stream-like structures associated with the LMC out to distances of $30^{\circ}$ from the LMC center, indicating that the area of sky that may contain Magellanic Cloud populations is truly enormous.

The picture that emerges from these recent discoveries is one in which the disk of the LMC is both much more extended than previously thought, and, particularly for the stellar populations in the periphery, much more disturbed. Clearly, the interaction between the Clouds (e.g., Besla et al. 2007, 2012) has left a strong imprint on the LMC's structure. The debris and distortions from tidal interactions is a key observational probe of the dynamical masses, orbits, and interaction histories of the galaxies. However, a complete understanding of the origin of these substructures requires a determination of the full extent and shape of the LMC stellar disk as well as other populations in the periphery.

Here, we use deep SMASH photometry of old mainsequence turnoff (MSTO) stars to probe the spatial distribution and origin of stellar populations in the LMC periphery to lower surface brightnesses than previously possible. We detect very extended stellar populations in many directions to $R \sim 21^{\circ}$ or $18.5 \mathrm{kpc}$ (at the LMC distance) with surface brightnesses as low as $\approx 34 \mathrm{mag} \operatorname{arcsec}^{-2}$ in the $g$-band. The layout of this paper is as follows. Section 2 gives an overview of our observations and data reduction. Section 3 describes the analysis of our deep color-magnitude diagrams, and the results are presented in Section 4. The relevance and interpretation of our measurements are discussed in Section 5, and, finally, our conclusions are summarized in Section 6.

\section{Observations and Data Reduction}

The SMASH survey is an NOAO community survey that used $\sim 50$ nights with the DECam on the CTIO Blanco $4 \mathrm{~m}$ telescope to perform deep imaging in ugriz of $~ 200$ "island" fields spread over $\sim 2400 \mathrm{deg}^{2}$ of the southern sky, resulting in an extended map with $\sim 20 \%$ filling factor. In the inner regions of the Clouds, the observed fields fully overlap, yielding a filled map of the main bodies. Figure 1 shows the region around the Magellanic Clouds and the SMASH fields used in the current analysis. These 21 fields include all deep SMASH fields with measurable LMC populations beyond the main disk body (where the stellar populations are more complicated) but excludes heavily dust obscured fields.

The survey observations and data reduction are fully described in Nidever et al. (2017). In brief, we used the InstCal image data products (calibrated, single-frame images) produced by the DECam Community Pipeline (CP; Valdes et al. 2014) and provided by the NOAO Science Archive Server. ${ }^{24}$ The photometric measurements were performed with the DAOPHOT (Stetson 1987, 1994) suite-based PHOTRED ${ }^{25}$ pipeline (Nidever et al. 2017). PHOTRED was used to perform WCS fitting, single-image PSF photometry (using ALLSTAR), forced PSF photometry of multiple images with a master source list created from a deep stack of all exposures (using ALLFRAME), aperture correction, photometric calibration, and dereddening. The photometric reduction resulted in measurements with median $5 \sigma$ depths of 23.9, 24.8, 24.5, 24.2, and $23.5 \mathrm{mag}$ in ugriz, respectively.

We also use the NOAO Source Catalog (NSC; Nidever et al. 2018) that contains aperture photometry of nearly all public DECam data including many SMASH exposures to select LMC MSTO stars and contiguously map the regions around the LMC (see Figure 1) with an eye to using both the SMASH and NSC data sets to further understand the structure of LMC. The NSC selection is $0.0 \leqslant g-r \leqslant 0.4$ and $21.8 \leqslant g \leqslant 22.8$ with shape FWHM $<1$ ". 5 to remove background galaxies. While the photometry was not dereddened, regions with SFD98 $E(B-V)>0.5 \mathrm{mag}$ were excluded.

\section{Analysis}

We conducted an analysis of the deep SMASH CMDs to measure the surface density of Magellanic stars using the MSTO. The portion of the CMD populated with these stars is contaminated by both background galaxies and foreground MW stars. The galaxies were removed with both color-color and morphological cuts while MW models were used to subtract the MW stars. Then, the LMC MSTO luminosity function was compared to a fiducial field to measure the surface density. The steps are described in more detail below.

\subsection{Removing Background Galaxies}

At faint magnitudes ( $g \gtrsim 23)$, there is a large number of galaxies that contaminate our stellar signal of the LMC MSTO (e.g., Fadely et al. 2012). Applying cuts on the morphological parameters only partially mitigates the problem because many of the galaxies are unresolved. To further cull the stellar sample, we apply cuts taking advantage of the limited region of the multi-dimensional color space that stars occupy (i.e., the stellar "locus") and the multi-band ugriz SMASH photometry. In each independent color-color diagram, bright PSF-like sources are used to determine the stellar locus (and its intrinsic width) in that plane. We use the $g-z, r-z$, and $i-z$ colors

\footnotetext{
24 https://www.portal-nvo.noao.edu

25 https://github.com/dnidever/PHOTRED
} 

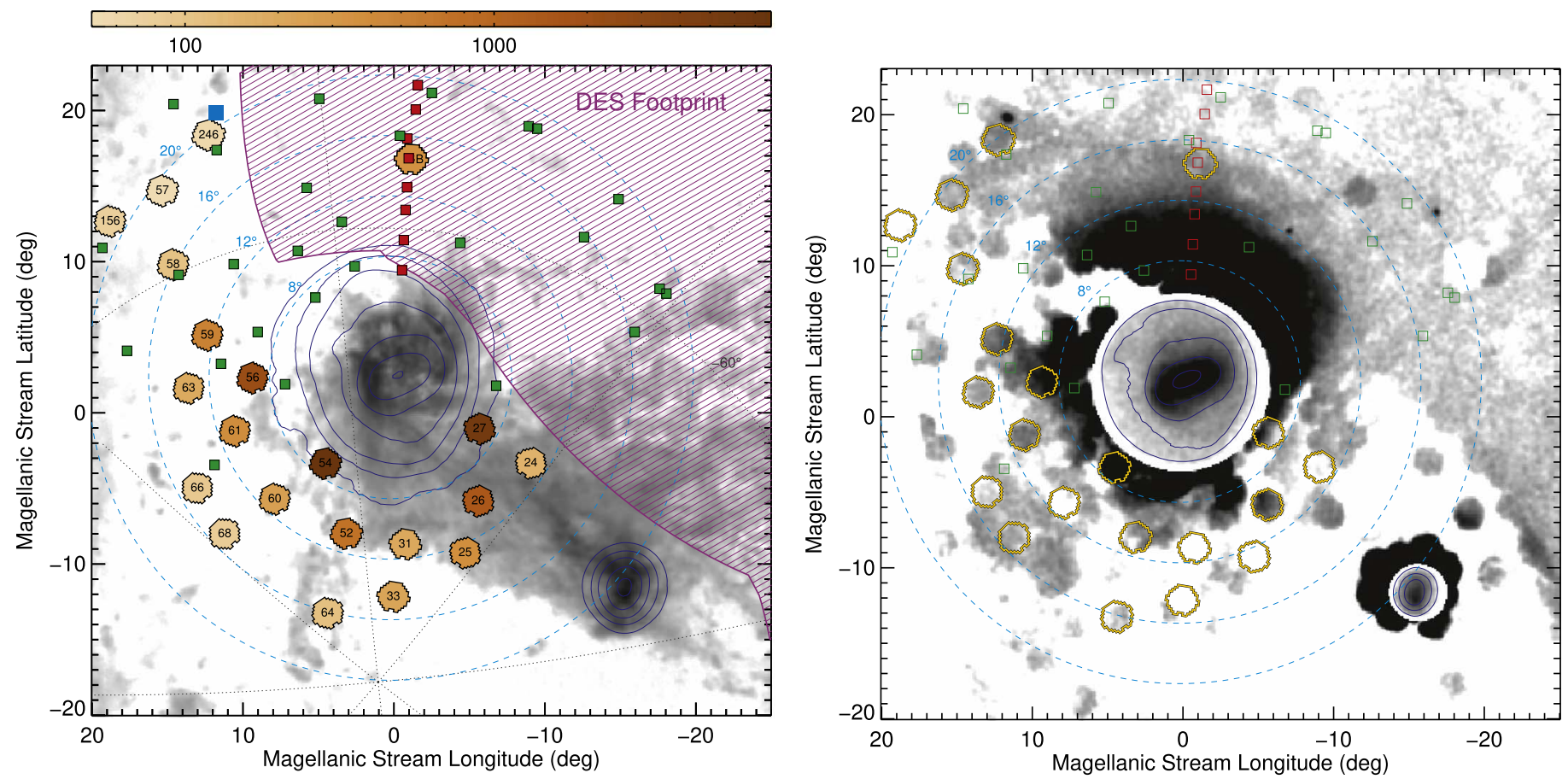

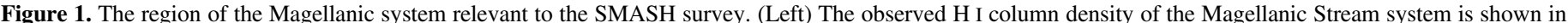

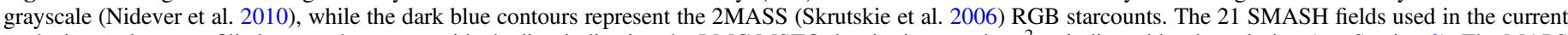

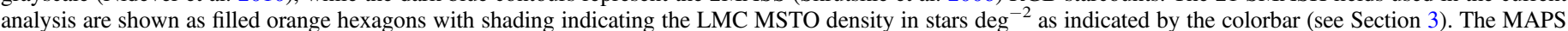

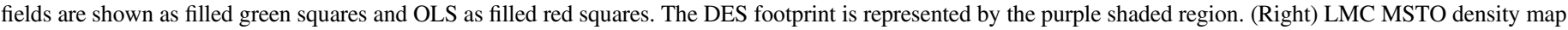

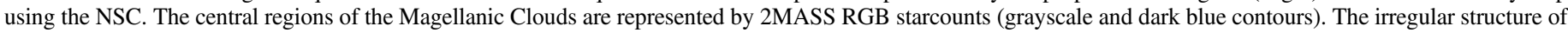

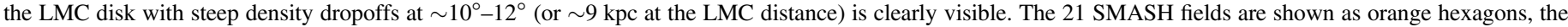
MAPS fields as open green squares, and the OLS fields as open red squares.

and $g-i$ as the fiducial color. The $u$-band was not used since it is not as deep as the other bands, and colors using $u$ show higher intrinsic scatter (especially for bluer objects) making it more challenging to remove galaxies as "outliers" in these colors. The stellar locus model is computed for each object (with good photometry in all three bands of a given color-color plane) using its $g-i$ color. If the deviation from the stellar locus is more than 2.5 times the observational color uncertainty or $0.2 \mathrm{mag}$ then the object is considered inconsistent with the stellar locus and removed. A morphological shape cut is also applied using the DAOPHOT SHARP parameter (Stetson 1987), which is a measure of the peakedness of an object's spatial profile compared to the PSF, with stars having values around zero while extended objects have large values. We set a lower cutoff of 0.2 on $\mid$ SHARP $\mid$ for the brighter stars and allow the threshold to grow slightly with magnitude but set an upper limit of 1.0. Finally, we also set a lower cutoff of 0.2 on the Source Extractor (Bertin \& Arnouts 1996) stellar probability value ( $\mathrm{PROB}$ column in the SMASH catalogs) but allow an exception for bright stars that have an artificially low $P R O B$ value likely due to the brighter-fatter effect (Gruen et al. 2015). An example of the stellar locus and morphology cuts is shown in Figure 2.

\subsection{Artificial Star Tests}

Artificial star tests (ASTs) were performed for all SMASH fields to estimate completeness. In brief, artificial stars uniformly spanning the $(g-i, g)$ CMD space $(-1.0<g-i<3.5$, $17.0<g<27.0$ ) were injected into all images for a single $\mathrm{CCD}$ and the images were processed with PHOTRED the same way the original images were. The final catalog of sources was then cross-matched with the original list of injected artificial stars. See Choi et al. (2018a) for a more detailed description of the SMASH single-chip AST procedure. Completeness maps were generated for the CMD analysis using the requirement that an AST had to be detected in at least one image in both $g$ and $i$ and pass the star/galaxy separation cuts (Figure 3).

\subsection{Cleaning Milky Way Populations}

MW populations are an important contaminant for our study of the MCs. Popular MW models such as the Besançon Galactic Model (Robin et al. 2003) or Galaxia (Sharma et al. 2011) provide unsatisfactory results for our deep SMASH data that probe regions of the CMD not previously used to constrain the existing models. Therefore, we fit our own MW model to the SMASH data itself. Spatial and population parameters for the MW (see Robin et al. 2003 for more details) were constrained by fitting the observed distribution of stars in a set of CMDs. To obtain the best constraint on the parameters of the different MW components, as large an area as possible was included in the fit. SMASH fields were selected in the southern hemisphere far from the MW midplane $\left(|b|>20^{\circ}\right)$ and the MCs ( $>10^{\circ}$ from each) to avoid populations not included in the model. The set of 38 fields were fit in the $(g, g-i)$ plane using Dartmouth isochrones $^{26}$ (Dotter et al. 2008) in the region $-0.2<g-i<1.8$ and $14<g<22.5$ to sample an area that excludes faint M-dwarfs (which are challenging to model) and where the data are nearly $100 \%$ complete. The resulting best-fit parameters for the thin disk, thick disk, and halo are mostly consistent with previous studies. Most importantly to our present work, the best fit to the data gives an MW halo

\footnotetext{
${ }^{26}$ http://stellar.dartmouth.edu/models/
} 

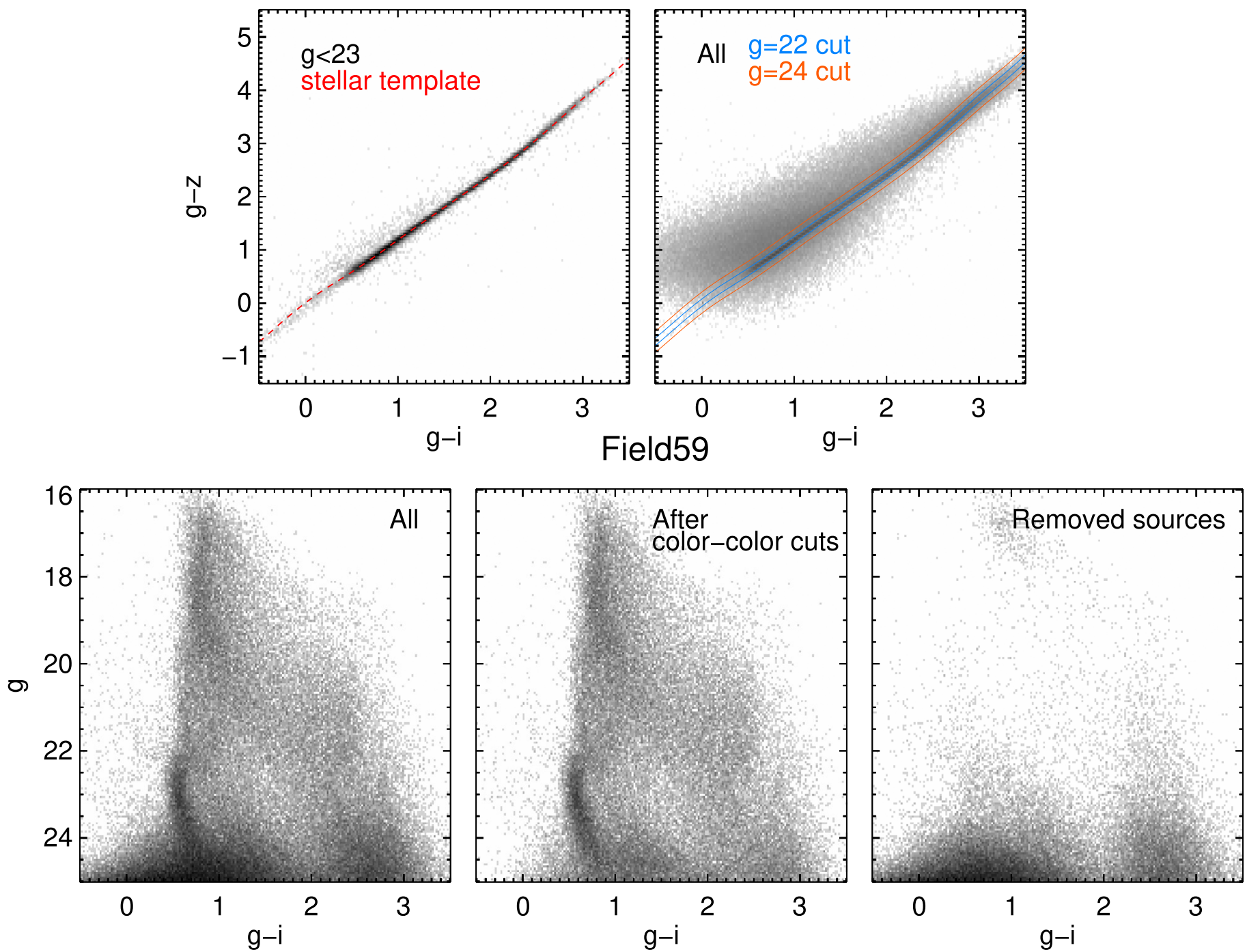

Figure 2. Illustration of our star/galaxy separation using the stellar locus in one color-color space for Field59 (three color-color combinations are used for the final cuts). Morphological shape and detection cuts ( $\mid$ SHARP $\mid<1$, CHI $<2$, and NDET $>5$ ) are already applied for the data in the figure. (Top left) $g-z$ vs. $g-i$ for $g<23.0$. The brighter sources are dominated by stars and are used to define the empirical template of the stellar locus (red dashed line). (Top right) $g-z$ vs. $g-i$ for all stars. The cuts around the stellar locus applied to objects at $g=22$ and $g=24 \mathrm{mag}$ are shown (blue and orange, respectively). (Bottom left) $g$ vs. $g-i$ CMD for all sources, (bottom middle) sources that pass all color-color stellar locus cuts such as shown in the top panel, and (bottom right) the removed sources. The large majority of extragalactic contaminants are removed.

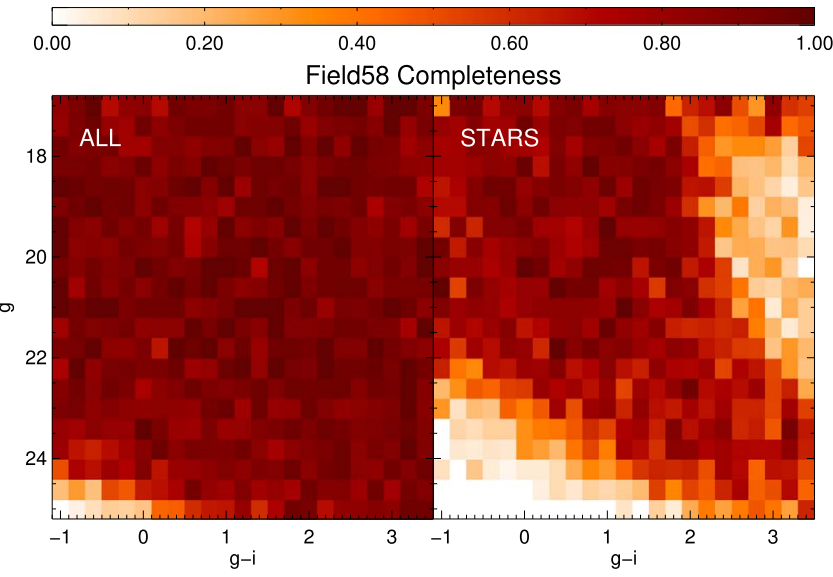

Figure 3. Completeness maps for Field58 for all objects (left) and after the star/galaxy cuts are applied (right) component that follows a power-law index of -3 and flattening $\sim 0.5$ similar to those seen previously (Bell et al. 2008; Deason et al. 2011; Slater et al. 2016). A more detailed description of this work will be presented in the near future (T. de Boer et al. 2019, in preparation). MW models were generated for each SMASH field using the best-fit parameters, and convolved with photometric error and completeness of each individual field.

The SMASH stellar catalogs use Schlegel et al. (1998, hereafter SFD98) $E(B-V)$ dereddening by default. However, CMDs of SFD98-dereddened photometry still showed slight field-to-field color offsets in the blue edge of the MW populations. Therefore, we recomputed field-specific reddening using color offsets of the MW blue edge. The edge was measured in the magnitude range $21.0<g<22.0$ for the MW model, the fiducial field, and the science field. The color offset relative to the MW model was then used as the $E(g-i)$ reddening and used to 

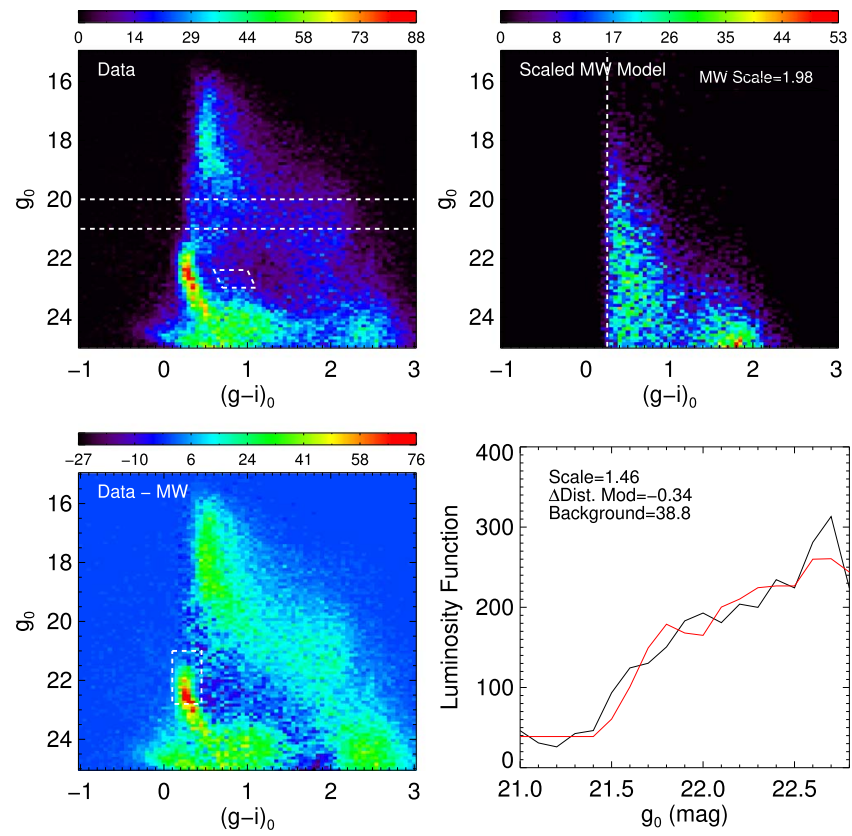

Figure 4. The CMD analysis for a single SMASH field (Field59). (Top left) Hess diagram of dereddened SMASH stars. The magnitude range used to determine the MW blue edge and the MW halo scaling box are shown in dashed lines. (Top right) Scaled MW halo density map and the measured blue edge (dashed line). (Bottom left) Observed Hess diagrams with the scaled MW halo subtracted. The box used to compute the luminosity function is shown in dashed lines. (Bottom right) Observed LMC MSTO luminosity function (black) and the scaled, shifted and offset model using the fiducial field (red).

compute the $A(g)$ extinction (with Schlafly \& Finkbeiner 2011 extinction coefficients) for the fiducial and science data.

Of the three MW populations (thin disk, thick disk, halo) only halo stars are present in the region of the CMD populated by the LMC MSTO. The halo component of the MW models was sometimes underrepresented (possibly because the faintest magnitudes were not included in the MW fitting) and we used a scaling factor to match the data. A parallelogram in the region $0.60 \leqslant(g-i)_{0} \leqslant 1.1$ and $22.4 \leqslant g_{0} \leqslant 23.0$ (see top left panel of Figure 4) was used to measure the observed and MW model densities and to calculate the MW halo scaling factor with a median value of 1.88 . The Hess density map in the dereddened CMD was generated for the observed SMASH stars and then the scaled MW halo model population map (corrected for completeness) was subtracted. It was more challenging to remove the MW halo component in the fields nearer to the MW midplane because of the presence of the Monoceros ring stars in the foreground (at $\sim 10-20 \mathrm{kpc}$ ). For these fields, the MW halo selection box was fine tuned for each field, and sometimes the MW halo scaling parameter was manually set after by-eye inspection of the residual image.

\subsection{Main-sequence Luminosity Functions}

For our CMD analysis, we use one SMASH field, FieldB north of the LMC (see Figure 1), with a well-measured LMC main sequence and good photometry with little extinction as our "fiducial" LMC field (see Figure 5). FieldB is at $R=14^{\circ} 5$, and the data were obtained during the SMASH pilot project and is therefore a magnitude deeper than the regular SMASH data.

We used the LMC main-sequence luminosity function to measure the density of LMC stars relative to the fiducial field. The region $0.11 \leqslant(g-i)_{0} \leqslant 0.44$ and $21.0 \leqslant g_{0} \leqslant 22.8$ (slightly fainter for the fiducial field) was used to measure the observed luminosity function, which extends somewhat brighter of the LMC MSTO to include a region to set the "background" level. The luminosity function was modeled using a shifted, scaled, and offset version of the fiducial luminosity function multiplied by the completeness map using an automated routine to find the best fit (see Figure 4). The mean background level was $\sim 50$ stars per $0.1 \mathrm{mag}$ bin while the number of LMC MSTO stars per bin ranged from 25 to 3100. This technique was found to be more reliable than a twodimensional modeling method, which was more sensitive to the shape and position of the LMC main sequence and consistently underestimated the LMC densities. The density of MSTO stars was then constructed by multiplying the scaling of the fiducial luminosity function by the number of fiducial MSTO stars with $g \leqslant 22.8$ (1014 stars) and dividing by the $3 \mathrm{deg}^{2}$ field of view of DECam (see Figure 6). The same factors were multiplied by the uncertainty in the fiducial luminosity scaling from the fitting procedure to obtain the uncertainty in the density of MSTO stars. The $g$-band surface brightness for the fiducial FieldB was calculated to be $32.02 \mathrm{mag} \operatorname{arcsec}^{1}$ by comparing the number of MSTO stars to synthetic photometry from a $Z=0.002,8 \mathrm{Gyr}, 50 \mathrm{kpc}$ BaSTI isochrone (Pietrinferni et al. 2004). Surface brightnesses for the other fields were bootstrapped off of the FieldB value by using the luminosity function scaling value. Table 1 gives density and other information on the 21 fields used in this study.

\section{Results}

Figure 1 shows the LMC density distribution from both the SMASH data (left) as well as the NSC (right), which highlight different spatial features. The features described in Mackey et al. (2016) are prominent in the NSC map including the northern spur and its extension to the west as well as the sharp density dropoff on the western side of the LMC. In addition, the newly discovered tidal spurs in the southern LMC by Mackey et al. (2018) are also partly visible in the NSC image. Moreover, the NSC data reveal previously unexplored structures on the eastern and northeastern side of the LMC, although the photographic isophotes from Irwin (1991) showed similar features but at smaller radii. A fairly sharp edge exists on the western side (possibly giving rise to the large variations in the SMASH densities in this area) that is reminiscent of the dropoff on the eastern side seen by Mackey et al. The northern spur and similar low-density features are extended farther to the northeast (toward Carina) than compared to Mackey et al. (2016). Overall, the shape of the LMC at $\sim 11^{\circ}$ is quite striking and somewhat triangular.

The SMASH data are able to probe to larger radii than the NSC map. Figure 5 presents example Hess diagrams of objects passing the star/galaxy separation for six of the 21 SMASH fields used in this study of the LMC periphery moving radially outward with the best "by-eye" fit isochrones. The LMC lower main sequence is clearly visible in all fields indicating that the deep SMASH data can be used to trace Magellanic populations to very low surface brightnesses.

Figure 6 shows the radial density profile of the LMC from individual SMASH (open hexagons), MAPS (small filled circles), and OLS (open circles) fields. Each symbol is colorcoded by its position angle. Both the SMASH and OLS densities were put on the MAPS scale of giants stars per $\mathrm{deg}^{2}$ using overlapping fields that have well-measured values. While the three data sets cover slightly different regions of the sky, 

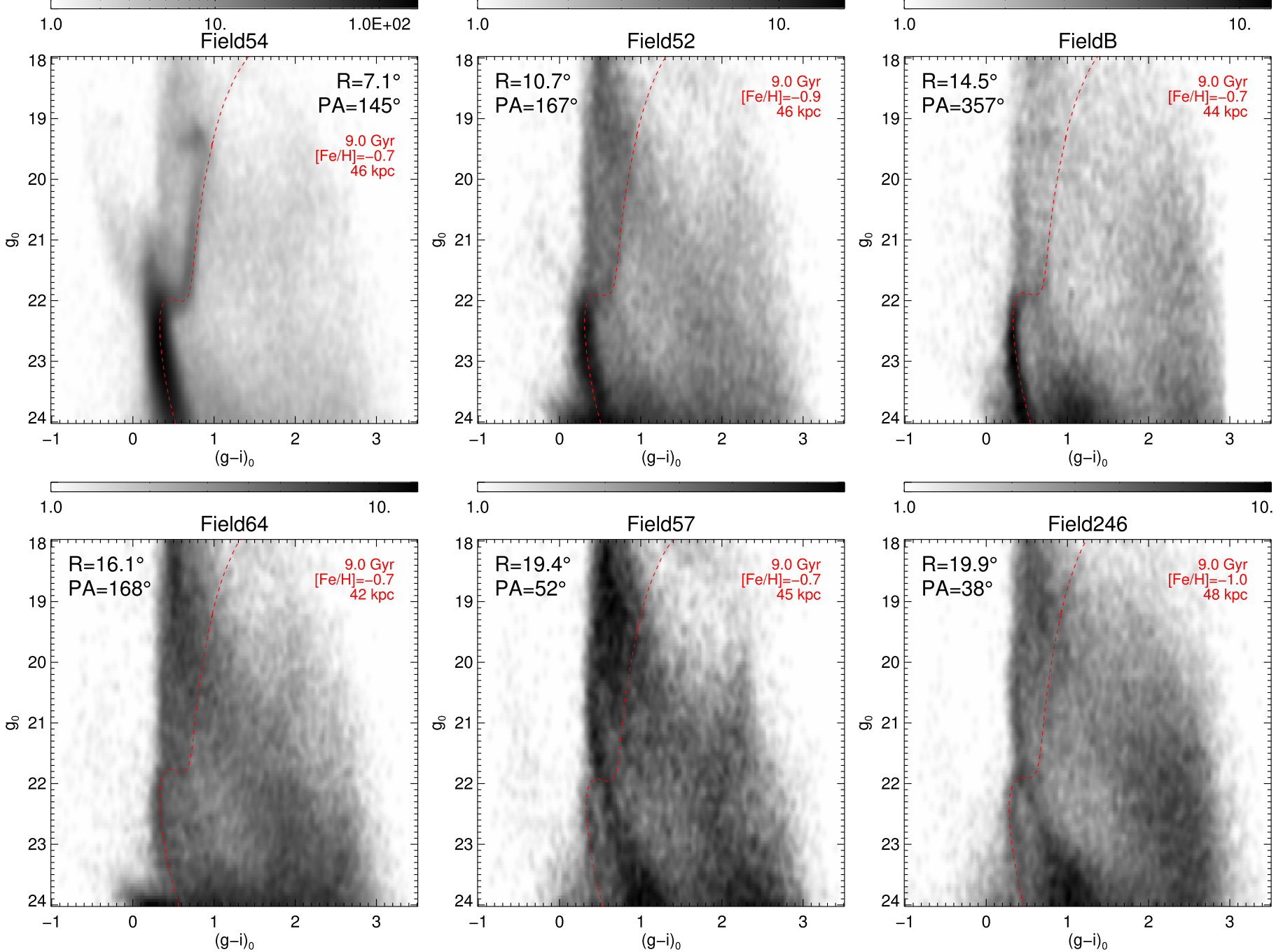

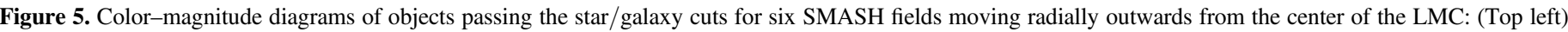

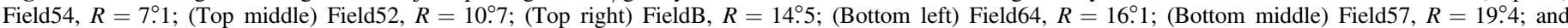

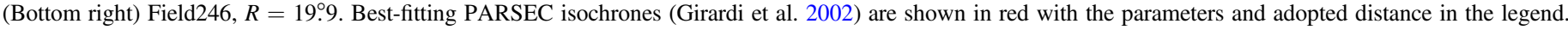

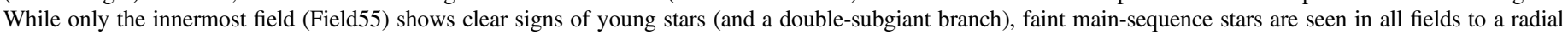
distance of 21.1 or $18.4 \mathrm{kpc}$ at the LMC distance.

their results generally agree with each other. All of them show an exponential decline out to $\sim 15^{\circ}$ with a scale-length of $\sim 1^{\circ}$. Both the MAPS and SMASH data show the existence of lowdensity populations beyond $\sim 16^{\circ}$ extending to $\sim 21^{\circ}$ with the SMASH best-fit inner exponential $\left(h_{\mathrm{R}}=1.1 \pm 0.03\right)$ and outer power-law $(\alpha=-2.2 \pm 0.4)$ exhibiting a "break" at $\sim 13^{\circ}-15^{\circ}$. The SMASH results show a significant scatter of $62 \%$ around the best-fit model (i.e., standard deviation of data/ model), especially as a function of position angle, with some fields deviating by as much as $2-2.5 \times$ from the best-fit model. Many of these variations are well above the uncertainties in the density measurements with $\sim 1 / 3$ of the points being $5 \sigma$ outliers resulting in the solution having a poor reduced $\chi^{2}=37.4$. At smaller radii, these variations are due to the inclined and intrinsically elongated LMC disk (van der Marel 2001), but at larger radii, this is likely because of the irregular shape of the LMC (as seen by Mackey et al. and the NSC data) as well as the substructure of spurs and stream-like features. The total mass of the power-low component (from $13^{\circ}$ to $23^{\circ}$ ), assuming it continues on the northwestern side not probed by SMASH, compared to the exponential component is
$0.4 \%$, although this quantity is quite uncertain due to the large variability in the LMC envelope.

\section{Discussion}

The origins of the stellar populations in the LMC periphery remain unclear. Majewski et al. (2009) proposed that the large spatial extent, radial profile, radial velocity profile, and low metallicity resembled an accreted stellar halo. In contrast, Saha et al. (2010) found that out to $\sim 16^{\circ}$, the radial profile (to the north) is well-fit by an exponential and, therefore, the stellar populations are likely an extended disk. Moreover, Mackey et al. (2016) claimed that the northern stream-like feature was material most likely stripped from the outskirts of the LMC disk by the MW's tidal force. Besla et al. (2016), Choi et al. (2018a, 2018b) discuss substructures in the LMC disk that likely arose due to the recent interactions with the SMC, and, potentially, these impulses could have created similar structures at larger radii.

The SMASH data reveal a very extended $\left(R \sim 21^{\circ}\right)$ "envelope" of old ( $\sim 9 \mathrm{Gyr})$ and relatively metal-poor 


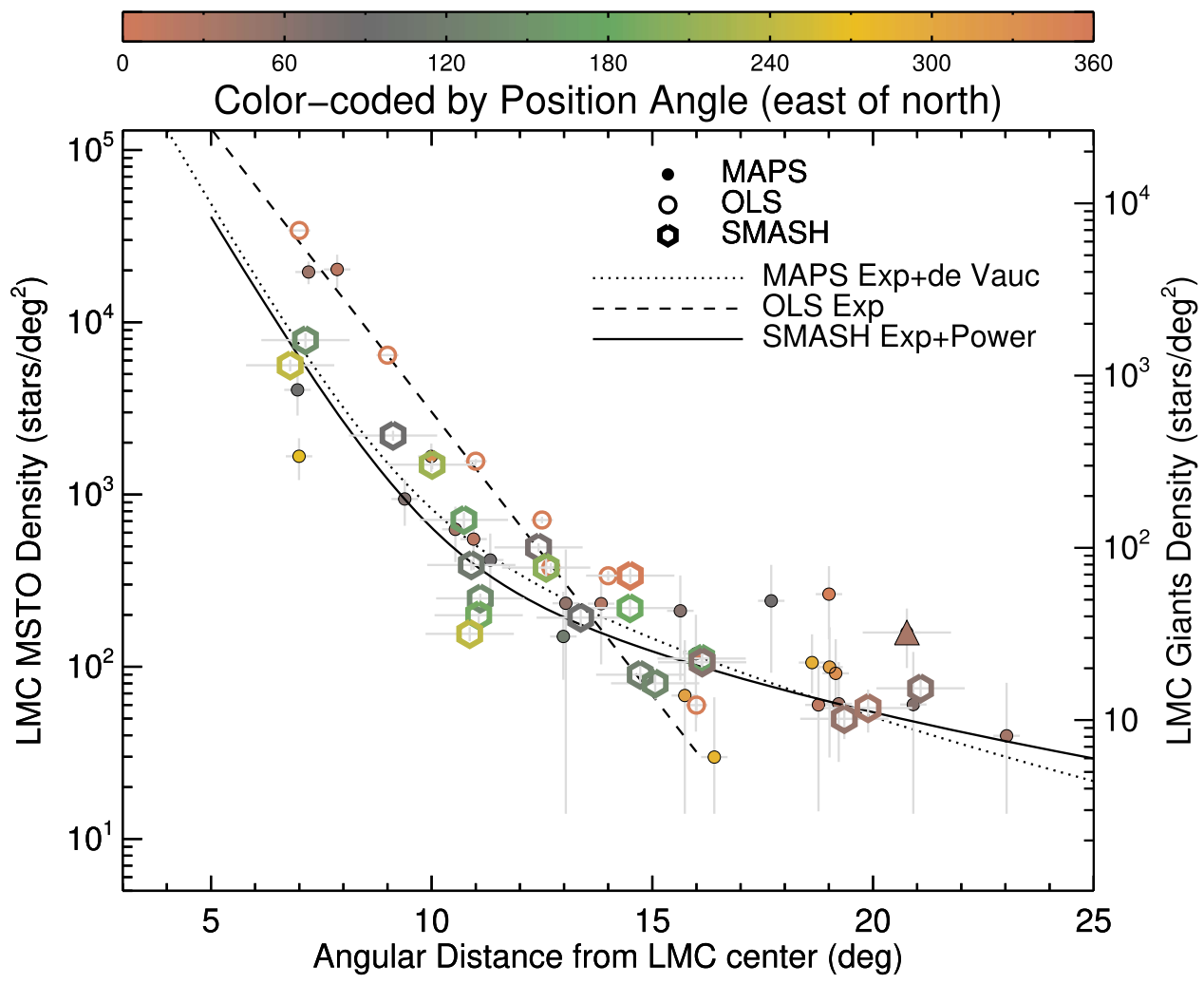

Figure 6. The density of LMC stars vs. radius for various studies. The MAPS fields are represented by small filled dots, open circles are data from OLS, and open hexagons are the SMASH fields. The group of LMC stars in the foreground of the Carina dwarf spheroidal discovered in Muñoz et al. (2006) is the large filled triangle. The points are color-coded by position angle (east of north). The density uncertainties are indicated by vertical gray lines while the radial coverage is indicated by the horizontal gray lines. The MAPS exponential plus de Vaucouleurs fit is shown as the dotted line, the OLS exponential fit is the dashed line, and the SMASH exponential plus power-law fit is the solid line.

$([\mathrm{Fe} / \mathrm{H}] \approx-0.8 \mathrm{dex})$ stars around the LMC covering a large range in position angle and possessing a shallow radial density profile at large radii that constitutes $\sim 0.4 \%$ of the LMC's stellar mass. Our results corroborate earlier work on the existence of this structure but add important empirical constraints using our deep CMDs especially in the south, east, and northeastern side of the LMC periphery. Our NSC map extends contiguous mapping to the eastern side of the LMC and uncovers an irregular shape and sharp density dropoff there $\left(\sim 10^{\circ}\right)$. This, combined with the Mackey et al. results, illustrates the pronounced asymmetric shape of the LMC at $\sim 10^{\circ}$. In addition, the SMASH densities exhibit large scatter around our best-fit exponential plus power-law model of $\sim 70 \%$. All of this shows that the structure of the LMC stellar periphery is highly disturbed and irregular, quite possibly due to tidal interactions with the SMC and MW.

The recent discovery of several dwarf satellite galaxies (e.g., Bechtol et al. 2015; Drlica-Wagner et al. 2015), many of them close to the MCs and some having radial velocities consistent with MC origins (e.g., Walker et al. 2016; Li et al. 2018), indicates that the MCs have their own system of satellites as suggested by simulations (Deason et al. 2015; Wheeler et al. 2015). It is therefore quite likely that the MCs also have a halo of disrupted satellite galaxies. We also note that the mass fraction that we derive for the LMC stellar envelope of $\sim 0.4 \%$ is quite similar to the mass fraction of the MW's stellar halo of 1\% (Carney et al. 1990; Bell et al. 2008) and expectations from simulations of stellar halos (e.g., Bullock \& Johnston 2005). In addition, RR Lyrae studies have found that the inner region of the LMC possesses a kinematically hot and very metal-poor $([\mathrm{Fe} / \mathrm{H}] \approx-1.5 \mathrm{dex})$ stellar component (Minniti et al. 2003; Borissova et al. 2004). Furthermore, recent SDSSIV APOGEE-2 (Blanton et al. 2017; Majewski et al. 2017) spectroscopic results also show the existence of a kinematically hot and metal-poor (to $[\mathrm{Fe} / \mathrm{H}] \approx-2.5 \mathrm{dex}$ ) population of RGB stars prominent at intermediate radii $\left(\sim 7^{\circ}-9^{\circ}\right.$; Nidever et al. 2019). Therefore, there is strong evidence from a variety of studies suggesting that an accreted halo of the LMC does exist and is roughly consistent with our observations of the LMC periphery.

On the other hand, the Magellanic interaction simulations of Besla et al. (2016) and Mackey et al. (2016) compellingly reproduce several substructures in the outer LMC. Tidally induced perturbations in the disk also create asymmetries and different density profiles in the north versus the south that can explain the observed higher densities in the northern portion of the LMC periphery. Moreover, the outer LMC disk is known to be old and somewhat more metal-poor compared to the inner regions (Carrera et al. 2008; Meschin et al. 2014), which could fit with the known characteristics of the periphery stars. This trend is also seen in simulations of dwarf galaxy formation that suggest they should have an extended distribution of old, metal-poor stars that resembles a "halo-like" component due to early star formation taking place in very low $V / \sigma$ gas (Read et al. 2006; El-Badry et al. 2018). In addition, the LMC disk is substantially thicker than those of larger spirals with a $V / \sigma=3$ (van der Marel et al. 2002), which is even lower than the MW's thick disk $(V / \sigma=4)$, making the distinction between a disk and halo component less clear. 
Table 1

SMASH LMC MSTO Densities

\begin{tabular}{lccrrrrr}
\hline \hline Field Name & $\begin{array}{c}\text { R.A. } \\
(\mathrm{J} 2000)\end{array}$ & $\begin{array}{c}\text { Decl. } \\
(\mathrm{J} 2000)\end{array}$ & $\begin{array}{c}R_{\text {LMC }} \\
(\mathrm{deg})\end{array}$ & $\begin{array}{c}\mathrm{PA}_{\text {LMC }} \\
(\mathrm{deg})\end{array}$ & $\begin{array}{c}\rho_{\text {MSTO }} \\
\text { stars deg }\end{array}$
\end{tabular}

Note.

${ }^{\mathrm{a}}$ The MSTO densities are calculated using the fiducial scaling factor multiplied by the number of MSTO stars with $g \leqslant=22.8$ (1014) divided by the DECam field of view $\left(3 \operatorname{deg}^{2}\right)$.

After the initial submission of this paper, the second data release from the Gaia mission (Gaia Collaboration 2018) has provided further insights into the outer stellar distribution of the LMC. Gaia only detects brighter stars than the faint mainsequence turnoff that we study here, but has the benefit of fullsky coverage and kinematical selection (e.g., with proper motions). The Gaia analysis of Belokurov \& Erkal (2019) confirms several of the findings presented here. For example, they also find that the LMC stellar populations extend $\sim 20^{\circ}$ to the south, east, and north while there is a lack of extended structure to the west. In addition, they find signs of substructure that are qualitatively consistent with the large scatter we detect. Finally, they find a stream-like or arm feature toward the south that, in combination with the northern arm, lends support to an interaction-induced origin of the outer stellar structures.

Therefore, it is quite likely that both a tidally disturbed disk (with a potentially old, metal-poor extended component) as well as the accretion of satellites (e.g., producing a classical halo) contribute to the stellar envelope that we reveal here, but further information (e.g., stellar kinematics and abundances) will be needed to fully unravel their relative contributions.

\section{Summary}

We have used deep SMASH photometry obtained with CTIO-4 $\mathrm{m}$ Blanco and DECam to study the low surface brightness features in the periphery of the Large Magellanic Cloud. Our data reveal a very extended stellar envelope around the LMC reaching to large radii with a distorted structure. Our main conclusions are:

1. We detect faint LMC main-sequence populations to very large radii in many directions reaching $R=21^{\circ}$. 1 in the northeast with a surface brightness of $\Sigma_{g} \approx 34 \mathrm{mag}$ $\operatorname{arcsec}^{2}$.
2. The deep SMASH CMDs of the outer LMC reveal the stellar populations to be old $(\sim 9 \mathrm{Gyr})$ and relatively metal-poor $([\mathrm{Fe} / \mathrm{H}] \approx-0.8 \mathrm{dex})$.

3. The LMC surface density profile initially follows an exponential decline $\left(h_{\mathrm{R}}=1.1 \pm 0.03\right)$ but "breaks" to a shallower slope at $\sim 13^{\circ}-15^{\circ}$ with a power-law index of $\alpha=-2.2 \pm 0.4$. The low-density outer component $\left(13^{\circ} \leqslant R \leqslant 23^{\circ}\right.$ ) contributes $\sim 0.4 \%$ of the total stellar mass of the LMC.

4. The SMASH main-sequence densities show large azimuthal variations with a scatter of $\sim 70 \%$ around the the best-fit model, but with some deviating by $2-2.5 \times$ from the average density. This shows that the structure of the outer LMC populations is highly distorted.

5. Using LMC main-sequence turnoff stars from the NOAO Source Catalog, we find a steep dropoff in density on the eastern side at $R \approx 8^{\circ}$, similar to the dropoff seen by Mackey et al. (2016) on the western side, as well as a low-density feature extending far to the northeast.

The origin of the outer LMC stellar envelope remains unclear with evidence for both an accreted halo and tidal stripping of the outer disk. Additional information such as accurate distances and line-of-sight depths, kinematics, and chemical abundances will be useful in unraveling the mechanisms responsible for creating this extensive structure. Finally, the low surface brightness measurements presented here should provide quantitative constraints on simulations of the formation of dwarf galaxies as well as the interaction of the Magellanic Clouds that will help improve our understanding of these important systems.

Y.C. \& E.F.B. acknowledge support from NSF grant AST 1655677. T.d.B. acknowledges financial support from the ERC 
under grant Agreement n. 308024 . B.C.C. acknowledges the support of the Australian Research Council through Discovery project DP150100862. M.-R.C. acknowledges support from the European Research Council (ERC) consolidator grant programme No. 682115. C.G. and M.M. acknowledge support by the Spanish Ministry of Economy and Competitiveness (MINECO) under the grants AYA2014-56795-P and AYA2017-89076-P. R.R.M. acknowledges partial support from project BASAL AFB-170002 as well as FONDECYT project $\mathrm{N}^{\circ} 1170364$. G.S.S. is supported by grants from NASA. We thank the anonymous referee for useful comments that improved the manuscript.

Based on observations at Cerro Tololo Inter-American Observatory, National Optical Astronomy Observatory (NOAO Prop. ID: 2013A-0411 and 2013B-0440; PI: Nidever), which is operated by the Association of Universities for Research in Astronomy (AURA) under a cooperative agreement with the National Science Foundation. This project used data obtained with the Dark Energy Camera (DECam), which was constructed by the Dark Energy Survey (DES) collaborating institutions: Argonne National Lab, University of California Santa Cruz, University of Cambridge, Centro de Investigaciones Energeticas, Medioambientales y Tecnologicas-Madrid, University of Chicago, University College London, DES-Brazil consortium, University of Edinburgh, ETH-Zurich, University of Illinois at Urbana-Champaign, Institut de Ciencies de l'Espai, Institut de Fisica d'Altes Energies, Lawrence Berkeley National Lab, Ludwig-Maximilians Universität, University of Michigan, National Optical Astronomy Observatory, University of Nottingham, Ohio State University, University of Pennsylvania, University of Portsmouth, SLAC National Lab, Stanford University, University of Sussex, and Texas A\&M University. Funding for DES, including DECam, has been provided by the U.S. Department of Energy, National Science Foundation, Ministry of Education and Science (Spain), Science and Technology Facilities Council (UK), Higher Education Funding Council (England), National Center for Supercomputing Applications, Kavli Institute for Cosmological Physics, Financiadora de Estudos e Projetos, Fundação Carlos Chagas Filho de Amparo a Pesquisa, Conselho Nacional de Desenvolvimento Cient?fico e Tecnológico and the Ministério da Ciência e Tecnologia (Brazil), the German Research Foundation-sponsored cluster of excellence "Origin and Structure of the Universe" and the DES collaborating institutions.

\section{ORCID iDs}

David L. Nidever (1) https://orcid.org/0000-0002-1793-3689

Knut Olsen (i) https://orcid.org/0000-0002-7134-8296

Yumi Choi (i) https://orcid.org/0000-0003-1680-1884

Thomas J. L. de Boer (1) https://orcid.org/0000-00015486-2747

Robert D. Blum (1) https://orcid.org/0000-0002-8622-4237

Eric F. Bell (1) https://orcid.org/0000-0002-5564-9873

Dennis Zaritsky (1) https://orcid.org/0000-0002-5177-727X

Nicolas F. Martin (1) https://orcid.org/0000-0002-1349-202X

Abhijit Saha (1) https://orcid.org/0000-0002-6839-4881

Blair C. Conn 주 https://orcid.org/0000-0001-6959-4546

Roeland P. van der Marel (i) https://orcid.org/0000-00017827-7825

Antonela Monachesi (i) https://orcid.org/0000-0003-

2325-9616

Guy S. Stringfellow ํㅏ https://orcid.org/0000-0003-1479-3059
Maria-Rosa L. Cioni (i) https://orcid.org/0000-00026797-696X

Carme Gallart (1) https://orcid.org/0000-0001-6728-806X

Matteo Monelli (i) https://orcid.org/0000-0001-5292-6380

David Martinez-Delgado (i) https://orcid.org/0000-0003-

3835-2231

Steven R. Majewski i https://orcid.org/0000-0003-

2025-3147

A. Katherina Vivas (ib https://orcid.org/0000-0003-4341-6172

Alistair R. Walker (10 https://orcid.org/0000-0002-7123-8943

You-Hua Chu (1) https://orcid.org/0000-0003-3667-574X

\section{References}

Bechtol, K., Drlica-Wagner, A., Balbinot, E., et al. 2015, ApJ, 807, 50 Bell, E. F., Zucker, D. B., Belokurov, V., et al. 2008, ApJ, 680, 295

Belokurov, V., Erkal, D., Deason, A. J., et al. 2017, MNRAS, 466, 4711

Belokurov, V., Irwin, M. J., Koposov, S. E., et al. 2014, MNRAS, 441, 2124

Belokurov, V., \& Koposov, S. E. 2016, MNRAS, 456, 602

Belokurov, V. A., \& Erkal, D. 2019, MNRAS, 482, L9

Bertin, E., \& Arnouts, S. 1996, A\&AS, 117, 393

Besla, G., Kallivayalil, N., Hernquist, L., et al. 2007, ApJ, 668, 949

Besla, G., Kallivayalil, N., Hernquist, L., van der Marel, R. P., Cox, T. J., \& Kereš, D. 2010, ApJL, 721, L97

Besla, G., Kallivayalil, N., Hernquist, L., van der Marel, R. P., Cox, T. J., \& Kereš, D. 2012, MNRAS, 421, 2109

Besla, G., Martínez-Delgado, D., van der Marel, R. P., et al. 2016, ApJ, 825, 20 Blanton, M. R., Bershady, M. A., Abolfathi, B., et al. 2017, AJ, 154, 28 Borissova, J., Minniti, D., Rejkuba, M., et al. 2004, A\&A, 423, 97

Brown, T. M., Tumlinson, J., Geha, M., et al. 2014, ApJ, 796, 91

Bullock, J. S., \& Johnston, K. V. 2005, ApJ, 635, 931

Carney, B. W., Latham, D. W., \& Laird, J. B. 1990, AJ, 99, 572

Carrera, R., Conn, B. C., Noël, N. E. D., Read, J. I., \& López Sánchez, Á. R. 2017, MNRAS, 471, 4571

Carrera, R., Gallart, C., Hardy, E., Aparicio, A., \& Zinn, R. 2008, AJ, 135, 836 Chapman, S. C., Ibata, R., Lewis, G. F., et al. 2006, ApJ, 653, 255

Choi, Y., Nidever, D. L., Olsen, K., et al. 2018a, ApJ, 866, 90

Choi, Y., Nidever, D. L., Olsen, K., et al. 2018b, ApJ, 896, 125

Dark Energy Survey Collaboration, Abbott, T., Abdalla, F. B., et al. 2016, MNRAS, 460, 1270

Deason, A. J., Belokurov, V., \& Evans, N. W. 2011, MNRAS, 416, 2903

Deason, A. J., Wetzel, A. R., Garrison-Kimmel, S., \& Belokurov, V. 2015, MNRAS, 453, 3568

de Vaucouleurs, G., \& Freeman, K. C. 1972, VA, 14, 163

D’Onghia, E., \& Lake, G. 2008, ApJL, 686, L61

Dotter, A., Chaboyer, B., Jevremović, D., et al. 2008, ApJS, 178, 89

Drlica-Wagner, A., Bechtol, K., Rykoff, E. S., et al. 2015, ApJ, 813, 109

El-Badry, K., Quataert, E., Wetzel, A., et al. 2018, MNRAS, 473, 1930

Epchtein, N., de Batz, B., Capoani, L., et al. 1997, Msngr, 87, 27

Fadely, R., Hogg, D. W., \& Willman, B. 2012, ApJ, 760, 15

Flaugher, B., Diehl, H. T., Honscheid, K., et al. 2015, AJ, 150, 150

Gaia Collaboration 2018, A\&A, 616, A1

Gardiner, L. T., \& Noguchi, M. 1996, MNRAS, 278, 191

Girardi, L., Bertelli, G., Bressan, A., et al. 2002, A\&A, 391, 195

Gómez, F. A., Besla, G., Carpintero, D. D., et al. 2015, ApJ, 802, 128

Gruen, D., Bernstein, G. M., Jarvis, M., et al. 2015, JInst, 10, C05032

Guhathakurta, P., Ostheimer, J. C., Gilbert, K. M., et al. 2005, arXiv:astro-ph/ 0502366

Irwin, M. J. 1991, The Magellanic Clouds, 148, 453

Li, T. S., Simon, J. D., Pace, A. B., et al. 2018, ApJ, 857, 145

Mackey, A. D., Koposov, S. E., Erkal, D., et al. 2016, MNRAS, 459, 239

Mackey, D., Koposov, S., Da Costa, G., et al. 2018, ApJL, 858, L21

Majewski, S. R., Nidever, D. L., Muñoz, R. R., et al. 2009, in IAU Symp. 256,

The Magellanic System: Stars, Gas, and Galaxies, ed. J. Th. van Loon \& J. M. Oliveira (Cambridge: Cambridge Univ. Press), 51

Majewski, S. R., Schiavon, R. P., Frinchaboy, P. M., et al. 2017, AJ, 154, 94 Martin, N. F., de Jong, J. T. A., \& Rix, H.-W. 2008, ApJ, 684, 1075

McConnachie, A. W., Chapman, S. C., Ibata, R. A., et al. 2006, ApJL, 647, L25

Meschin, I., Gallart, C., Aparicio, A., et al. 2014, MNRAS, 438, 1067

Minniti, D., Borissova, J., Rejkuba, M., et al. 2003, Sci, 301, 1508

Muñoz, R. R., Carlin, J. L., Frinchaboy, P. M., et al. 2006, ApJL, 650, L51

Muñoz, R. R., Geha, M., \& Willman, B. 2010, AJ, 140, 138

Nidever, D. L. 2009, PhD dissertation, Univ. Virginia 
Nidever, D. L., Dey, A., Olsen, K., et al. 2018, AJ, 156, 131

Nidever, D. L., Hasselquist, S., Hayes, C. R., et al. 2019, ApJ, submitted, (arXiv:1901.03448)

Nidever, D. L., Majewski, S. R., Butler Burton, W., \& Nigra, L. 2010, ApJ, 723, 1618

Nidever, D. L., Majewski, S. R., Muñoz, R. R., et al. 2011, ApJL, 733, L10

Nidever, D. L., Monachesi, A., Bell, E. F., et al. 2013, ApJ, 779, 145

Nidever, D. L., Olsen, K., Walker, A. R., et al. 2017, AJ, 154, 199

Noël, N. E. D., Conn, B. C., Carrera, R., et al. 2013, ApJ, 768, 109

Pietrinferni, A., Cassisi, S., Salaris, M., \& Castelli, F. 2004, ApJ, 612, 168

Putman, M. E., Staveley-Smith, L., Freeman, K. C., Gibson, B. K., \& Barnes, D. G. 2003, ApJ, 586, 170 (P03)

Read, J. I., Pontzen, A. P., \& Viel, M. 2006, MNRAS, 371, 885

Robin, A. C., Reylé, C., Derrière, S., \& Picaud, S. 2003, A\&A, 409, 523

Saha, A., Olszewski, E. W., Brondel, B., et al. 2010, AJ, 140, 1719

Sales, L. V., Navarro, J. F., Cooper, A. P., et al. 2011, MNRAS, 418, 648

Schlafly, E. F., \& Finkbeiner, D. P. 2011, ApJ, 737, 103
Schlegel, D. J., Finkbeiner, D. P., \& Davis, M. 1998, ApJ, 500, 525

Sharma, S., Bland-Hawthorn, J., Johnston, K. V., \& Binney, J. 2011, ApJ, 730,3

Skrutskie, M. F., Cutri, R. M., Stiening, R., et al. 2006, AJ, 131, 1163

Slater, C. T., Nidever, D. L., Munn, J. A., Bell, E. F., \& Majewski, S. R. 2016, ApJ, 832, 206

Stetson, P. B. 1987, PASP, 99, 191

Stetson, P. B. 1994, PASP, 106, 250

Valdes, F., Gruendl, R. \& DES Project 2014, in ASP Conf. Ser. 485, Astronomical Data Analysis Software and Systems XXIII, ed. N. Manset \& P. Forshay (San Francisco, CA: ASP), 379

van der Marel, R. P. 2001, AJ, 122, 1827

van der Marel, R. P., Alves, D. R., Hardy, E., \& Suntzeff, N. B. 2002, AJ, 124, 2639

van der Marel, R. P., \& Kallivayalil, N. 2014, ApJ, 781, 121

Walker, M. G., Mateo, M., Olszewski, E. W., et al. 2016, ApJ, 819, 53

Wheeler, C., Oñorbe, J., Bullock, J. S., et al. 2015, MNRAS, 453, 1305 